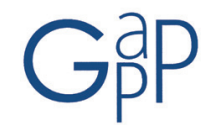

GESTIÓN Y ANÁLISIS DE POLÍTICAS PÚBLICAS, Nueva Época, - N.ํ15, enero-junio 2016 - ISSN: 1989-8991

DOI: http://dx.doi.org/10.24965/gapp.voi15.10314

Agustí Cerrillo i Martínez

Universitat Oberta de Catalunya

acerrillo@uoc.edu

\title{
Las compras abiertas y la prevención de la corrupción*
}

\section{Resumen}

La conexión de las políticas de gobierno abierto y la contratación pública ha dado lugar al surgimiento de las compras abiertas que constituyen un ámbito de investigación ciertamente novedoso. Este trabajo persigue exponer las contribuciones de las compras abiertas a la prevención y la lucha contra la corrupción. En primer lugar, se examina el reflejo de las compras abiertas en la legislación española en materia de contratación pública, de transparencia y de gobierno abierto. A continuación, se muestran las principales manifestaciones de la corrupción en la contratación pública y se analiza el impacto que las compras abiertas pueden tener en su prevención y denuncia. Finalmente, a partir de las carencias observadas en la regulación y en las prácticas que se han impulsado en España hasta el momento, se proponen los elementos que deberían incorporarse en la futura regulación de la contratación pública para facilitar que las compras abiertas puedan colaborar a la prevención y la lucha contra la corrupción.

Palabras clave

Compras abiertas; gobierno abierto; corrupción; regulación

\section{Open contracting and prevention of corruption}

\section{Abstract}

The connection between open government policies and public procurement has brought up open contracting as a new research field. This paper aims to explain how open contracting can contribute to prevent and fight against corruption. Firstly, the reflection of open contracting in public procurement, transparency and open government regulation is examined. Next, the main manifestations of corruption in public procurement are showed and the impact of open contracting in prevention and fighting against corruption is analyzed. Finally, from the shortcomings identified in Spanish regulation and in Spanish Public Administrations practices, there is a proposal of the elements that should be included in the future regulation of public procurement to facilitate that open contracting can collaborate to prevent and fight against corruption.

Keywords

Open contracting; open government; corruption; regulation.

* Trabajo enmarcado en el proyecto "Transparencia, Integridad e Innovación Democrática”. Programa Estatal de I+D+i Orientada a los Retos de la Sociedad (DER2014-57391-C2-2-R). Un versión previa de este trabajo fue presentada en el XX Congreso Internacional del CLAD sobre la Reforma del Estado y de la Administración Pública celebrado en Lima, del 10 al 13 de noviembre de 2015. 


\section{PRESENTACIÓN}

La contratación pública es uno de los ámbitos de la actividad pública más expuesto a los conflictos de intereses, los favoritismos y la corrupción por la estrecha interacción entre las administraciones públicas y los empresarios, los amplios márgenes de discrecionalidad existentes y el elevado volumen de recursos económicos en juego que la caracterizan. Al respecto, no podemos desconocer que tres de cada diez empresas en los Estados miembros de la Unión Europea (32\%) que participaron en una licitación creen que la corrupción les impidió adjudicarse el contrato (European Commission, 2014a, 87).

Las consecuencias de esta situación son catastróficas para el desarrollo económico y social puesto que la corrupción incrementa los costes de la contratación pública entre un 20\% y 25\% y en algunos casos hasta el 50\% (2008) (European Commission, 2014b, 21) hasta el extremo que, como ha calculado la consultora PricewaterhouseCoopers, los costes directos de la corrupción en la contratación pública en cinco sectores (carreteras y ferrocarriles; agua y residuos; obra pública; formación e I\&D) en ocho países europeos llegó a ser en 2010 entre 1.400 millones $€$ y 2.200 millones $€$ (PricewaterhouseCoopers \& Ecorys, 2013)

Ante este escenario, en los últimos años se han ido impulsando medidas muy diversas desde ámbitos distintos con la finalidad de frenar si no erradicar esta lacra de las sociedades contemporáneas que tan negativos efectos tiene en el desarrollo económico y social y en la calidad democrática de las instituciones.

Una de las medidas que ha de tener un impacto positivo con esta finalidad es el gobierno abierto con el que se persigue fortalecer las relaciones entre las administraciones públicas y la ciudadanía a través del fomento de la transparencia pública, la participación y la colaboración ciudadanas. En los últimos años, los principios del gobierno abierto se han ido concretando en diferentes ámbitos de la actividad administrativa y, en particular, en la contratación pública. Asimismo, se ha empezado a observar el impacto positivo que puede tener en la prevención y la lucha contra la corrupción.

En este trabajo nos proponemos analizar cuál puede ser el impacto que la apertura de la contratación pública puede tener en la prevención y la lucha contra la corrupción. Como veremos a continuación, la apertura de la contratación pública no se refiere únicamente a garantizar la igualdad y la competencia de los empresarios que participen en una licitación, sino particularmente a fortalecer la transparencia de los procedimientos de licitación para facilitar la participación y la colaboración ciudadana en la prevención y la lucha contra la corrupción en la contratación pública y, en última instancia, en la eficacia y eficiencia de las administraciones públicas. En particular, partimos de la hipótesis que la apertura de los datos relativos a las diferentes fases de la contratación pública puede fortalecer la participación y la colaboración ciudadana en la prevención y la lucha contra la corrupción.

En efecto, como tendremos oportunidad de observar en las próximas páginas, el gobierno abierto puede resultar un instrumento útil para prevenir y luchar contra la corrupción en la contratación pública a través de la difusión de datos relativos a la contratación en formatos que faciliten su reutilización y, por lo tanto, la participación ciudadana en la difusión y el análisis de dichos datos y, en última instancia, su colaboración en el monitoreo y la supervisión de la contratación pública que lleve a la identificación de situaciones que puedan esconder un caso de corrupción y, en su caso, a su denuncia ante las autoridades competentes para su investigación y/o sanción.

Para ello, en las próximas páginas, en primer lugar, analizaremos cuál es la incidencia del gobierno abierto en la contratación pública. A continuación, expondremos las manifestaciones del gobierno abierto en la legislación española lo que nos permitirá conocer el marco en el que se pueden impulsar estas medidas y cuál puede ser su reflejo en la contratación pública en España. Finalmente, valoraremos cuál puede ser el impacto del gobierno abierto en la prevención y la lucha contra la corrupción.

\section{EL GOBIERNO ABIERTO Y SU IMPACTO EN LA CONTRATACIÓN PÚBLICA}

En los últimos años se ha ido extendiendo en diferentes países la voluntad de fortalecer las relaciones entre las administraciones públicas y la ciudadanía a través de una profundización de la transparencia pública, la participación y la colaboración ciudadanas mediante el uso intensivo de la tecnología. El gobierno abierto persigue potenciar el diálogo constante entre la ciudadanía y las administraciones públicas para que la ciudadanía pueda conocer qué hacen las administraciones públicas y cómo lo hacen, las administraciones públicas puedan escuchar a la ciudadanía y adaptar sus decisiones a sus preferencias y, finalmente, cuenten con su colaboración en el desarrollo de las políticas públicas y en la prestación de los servicios públicos (Calderón \& Lorenzo, 2010).

El gobierno abierto ha sido objeto de gran reconocimiento e impulso en los últimos años, particularmente, desde la llegada de Barack Obama a la presidencia de los Estados Unidos de América y la adopción, en las primeras horas de su mandato en enero de 2009, del Memorándum sobre transparencia y gobierno abierto en el que se afir- 
maba el compromiso de su administración con la creación de un nivel sin precedentes de apertura en el gobierno a través de un sistema de transparencia, participación y colaboración para fortalecer la democracia y promover la eficiencia y la eficacia en el gobierno. De todos modos, a pesar de la importancia que ha adquirido en el último lustro el gobierno abierto, no podemos desconocer los esfuerzos que diferentes gobiernos habían hecho en el pasado para fortalecer la transparencia y acercarse a la ciudadanía particularmente como reacción frente diferentes escándalos políticos e institucionales que habían ido surgiendo señaladamente en las décadas de los 60 y 70 del siglo pasado (Ramírez-Alujas, 2011, 101); (Wirtz \& Birkmeyer, 2015, 382).

Sin embargo, el gobierno abierto no es un fenómeno exclusivamente norteamericano. En los últimos años encontramos numerosas iniciativas relativas al gobierno abierto tanto en Europa como en América Latina (Dassen \& Veyra, 2012) e incluso en otras latitudes como se puede observar al visitar la página web de la Open Government Partnership.. De hecho, como afirman Lee y Kwak "Open Government is entering a new phase and becoming an important global agenda" (Lee \& Kwak, 2012, 492).

El gobierno abierto tiene numerosas manifestaciones en la práctica de las administraciones públicas debido a su carácter multidimensional relacionado con la transparencia, la participación y la colaboración ciudadanas. El gobierno abierto persigue en primera instancia un fortalecimiento del principio democrático a través de una mayor rendición de cuentas por parte de las administraciones públicas, un mejor control de la ciudadanía sobre la actividad de las administraciones y, en última instancia, un mayor compromiso que permita aproximar las administraciones públicas a la ciudadanía reduciendo la lejanía con la que estas las perciben. En esta dirección, Villoria recuerda cómo el gobierno abierto es una exigencia de las democracias de calidad (Villoria Mendieta, 2010, 113). Pero más allá de estas finalidades, como tendremos oportunidad de mostrar con detalle en las próximas páginas, el gobierno abierto también puede tener un impacto significativo en la prevención y la lucha contra la corrupción.

El gobierno abierto se caracteriza por un uso intensivo de la tecnología que está teniendo un papel significativo en la extensión del gobierno abierto y en los diferentes mecanismos a través de los que este se manifiesta. En particular, debemos destacar la estrecha relación que existe entre el gobierno abierto con las tecnologías incluidas bajo la etiqueta de la web 2.0, lo que se ha conocido por algunos como el gobierno 2.0 (Chun, 2010), es decir, a la introducción en la gestión pública de los principios que inspiran la web 2.0 (Ramírez-Alujas, 2011, 102). Como se ha destacado, "[c]on el uso de la Web 2.0 (Government 2.0) y redes sociales, se persigue facilitar la comunicación, la "escucha activa" y la participación, aprovechar el conocimiento y la experiencia de los ciudadanos para ayudar en el diseño de políticas y provisión de servicios públicos, y la colaboración (en red) dentro y entre las administraciones públicas y más allá" (Cruz-Rubio, 2014, 11). Todo ello, se manifiesta en particular, en la prevención y la lucha contra la corrupción (Bertot, Jaeger, \& Grimes, 2010); (Davies \& Fumega, 2014, V).

Además, el desarrollo del gobierno abierto también está estrechamente vinculado a la apertura de datos (Corojan \& Campos, 2011, 7). En efecto, uno de los elementos que conforma el gobierno abierto es el relativo a los datos abiertos. La apertura de datos constituye una evolución de la reutilización de la información del sector público que fija su atención no tanto en garantizar las condiciones necesarias para que la información sea reutilizada como en que las administraciones públicas faciliten información que pueda ser fácilmente reutilizada. A través de los portales de datos abiertos las administraciones públicas ponen a disposición de la ciudadanía datos en formatos que permiten y facilitan su reutilización. A través de la reutilización de los datos, la ciudadanía puede innovar produciendo nuevas informaciones o servicios basados en los datos públicos pero también puede contribuir a incrementar la transparencia (Cerrillo i Martínez, 2012b). La apertura de datos constituye la base para el gobierno abierto en la medida en que sólo de esta manera a través de un acceso fácil y libre a la información del sector público se puede favorecer la participación y la colaboración (Parycek \& Sachs, 2010, 3).

Más allá de la extensión de los principios de transparencia, participación y colaboración y del uso de diferentes mecanismos que con carácter transversal persiguen garantizar su impacto en el conjunto de la actividad administrativa, encontramos diferentes manifestaciones sectoriales del gobierno abierto. La contratación pública es uno de dichos sectores donde ha surgido lo que se ha venido a llamar compras abiertas (open contracting). Las compras abiertas son una de las principales manifestaciones del gobierno abierto a nivel internacional.

La aplicación de los principios del gobierno abierto en la contratación pública se ha visto favorecida por diversas iniciativas a nivel internacional como la que ha impulsado el Banco Mundial quien ha reconocido que "[o]paque contracting processes are hindering development. Open Contracting seeks to address that through collective innovation and collaborative action" 2 o la Alianza para la Contratación Abierta (Open Contracting Partnership) cuyos objetivos

1 http://www.opengovpartnership.org/ (última visita: julio 2015).

2 https://wbi.worldbank.org/wbi/stories/open-contracting-growing-global-movement (última consulta: julio 2015). 
son la propuesta de normas internacionales; el fortalecimiento de la implementación de la contratación abierta y la promoción de la formación y la colaboración ${ }^{3}$.

Como se desprende de la lectura de los pocos documentos que existen sobre la materia a nivel internacional y del análisis de las experiencias pioneras que se han llevado a cabo, rápidamente se puede constatar que las compras abiertas son algo más que la contratación pública electrónica puesto que no persiguen sólo la incorporación de los medios electrónicos para facilitar una mayor eficacia y eficiencia de los procedimientos de contratación sino que también persiguen incrementar su transparencia a través de la difusión de una pluralidad de datos que permitan a la ciudadanía participar activamente en las licitaciones y controlar el desempeño de la actividad contractual por parte de las administraciones públicas lo que puede facilitar la colaboración ciudadana en la prevención y la lucha contra la corrupción.

Con la expresión compras abiertas se hace referencia al conjunto de reglas y prácticas establecidas para incrementar la transparencia, la participación y la colaboración en la contratación pública en todas sus fases (licitación, ejecución y justificación) (Marchessault, 2013):

- La transparencia se refiere básicamente a la difusión de información y datos que permitan un mejor conocimiento, seguimiento y control de la contratación pública.

- La participación se traduce, no tanto, en la presentación de proyectos a las licitaciones como en el uso de los datos difundidos para poder seguir, monitorear y analizar la actividad contractual.

- La colaboración se concreta en el retorno que la ciudadanía hace a la propia administración en función de la información y los datos conocidos y del uso que de los mismos haya hecho y se puede concretar, por ejemplo, en la denuncia o información sobre casos de corrupción o en la impugnación de determinadas decisiones que contravengan el marco de referencia en la contratación pública.

En los últimos años, en el marco de la Alianza para la Contratación Abierta se han impulsado los Principios Globales para las Contrataciones Abiertas. Estos principios se agrupan en dos grupos relativos a la divulgación proactiva y la participación, el monitoreo y la supervisión ${ }^{4}$ :

Divulgación proactiva

- Reconocimiento del derecho de acceso a información relacionada con la formación, adjudicación, ejecución, cumplimiento y terminación de los contratos públicos.

- La contratación pública se realizará en forma transparente y equitativa.

- Publicación oportuna, actual y sistemática de información suficiente relacionada con los contratos públicos.

a) Contratos.

b) Estudios previos, documentos de licitaciones, evaluaciones de desempeño de los contratos, garantías e informes de supervisión y auditoría.

c) Información referida a la formación del contrato (proceso de planeación; método de adjudicación y la justificación; alcance y especificaciones de cada contrato; criterio de evaluación y selección del licitante adjudicado; oferentes o participantes del proceso, los documentos que demuestran su elegibilidad y las excepciones que les son aplicables; cualquier conflicto de intereses revelado; resultados de la evaluación, incluyendo justificación de la adjudicación; e identidad del adjudicatario del contrato y cualquier declaración de titularidad real realizada).

d) Información relacionada con la ejecución y el cumplimiento de los contratos públicos (información sobre subcontrataciones, modificaciones y ampliaciones a los contratos.

- Desarrollo de sistemas para recolectar, administrar, simplificar y publicar datos referidos a la celebración, adjudicación, cumplimiento y terminación de contratos públicos en un formato abierto y estructurado, que presente los datos de forma amigable y que facilite su búsqueda, todo esto de conformidad con el Estándar de Datos de Contrataciones Abiertas.

- Información pública de los contratos completa, con las excepciones o limitaciones definidas por la ley.

3 http://www.open-contracting.org/ (última consulta: julio 2015).

4 http://www.open-contracting.org/principios_globales_para_las_contrataciones_abiertas (última consulta: julio 2015). GESTIÓN Y ANÁLISIS DE POLÍTICAS PÚBLICAS, Nueva Época - N. 15, enero-junio 2016 - ISSN: 1989-8991 - DOI: 10.24965/gapp.voi15.10314 
- Apoyo de la divulgación de la información de las contrataciones públicas evitando cláusulas de confidencialidad, aplicando la confidencialidad de manera restringida a fin de que abarque sólo las excepciones permitidas, o incluyendo disposiciones en las cláusulas y condiciones contractuales que permitan la divulgación del contrato y de la información relacionada.

Participación, monitoreo y supervisión

- Reconocimiento del derecho a participar en la supervisión de la formación, adjudicación, cumplimiento y terminación de contratos públicos.

- Fomento de un entorno favorable que reconozca, fomente, promocione, proteja y genere oportunidades de consulta y monitoreo por parte de la ciudadanía en relación a las contrataciones públicas.

- Fortalecimiento de las capacidades de todos estos actores, para comprender, monitorear y mejorar el desempeño de las contrataciones públicas, así como para crear mecanismos de financiación sostenibles que apoyen la participación en las contrataciones públicas.

- Obligación de asegurar a las agencias de control y a las entidades ejecutoras, formas de acceso y uso de la información divulgada. Las entidades deben recibir la retroalimentación de los ciudadanos, actúan con base en ella y fomentan el diálogo y consulta entre las partes contratantes y las organizaciones de sociedad civil a fin de mejorar la calidad de los resultados de las contrataciones.

- Estrategias de consulta ciudadana y de participación durante la ejecución del contrato en relación con los contratos de impacto significativo.

\section{CUADRO 1: PRINCIPIOS GLOBALES PARA LAS CONTRATACIONES ABIERTAS}

Como se desprende de la lectura del preámbulo de los Principios Globales para las Contrataciones Abiertas una amplía divulgación y participación en las contrataciones públicas contribuirá a que las contrataciones sean más competitivas y justas, mejorando el desempeño contractual y asegurando el logro de los resultados de desarrollo previstos. Los principios engloban tanto el reconocimiento del derecho de acceso a la información sobre contratación pública como la difusión proactiva de la información contractual respecto a la que se definen las características y el contenido mínimo que debe tener. Asimismo, persiguen facilitar la participación y la colaboración ciudadana tanto en la ejecución de la contratación pública como en el seguimiento y supervisión de la actividad contractual de las administraciones públicas.

Estos principios deben inspirar a todos los actores que participan en la contratación pública. Sin embargo, carecen de carácter coercitivo por lo que su eficacia puede verse muy limitada mientras los estados no los incorporen en su ordenamiento jurídico. Como tendremos oportunidad de observar en el epígrafe siguiente, la legislación española incorpora algunas de las obligaciones que se derivan de los Principios Globales para las Contrataciones Abiertas particularmente desde la perspectiva de la transparencia, pero no promueve la participación, monitoreo y supervisión ciudadana de la contratación pública a través de los mecanismos que prevén los Principios Globales para las Contrataciones Abiertas por lo que la eficacia de estos principios y, en general, del gobierno abierto en la contratación pública puede verse muy limitada.

\section{LAS COMPRAS ABIERTAS EN LA LEGISLACIÓN ESPAÑOLA}

El gobierno abierto y su manifestación en la contratación pública, las compras abiertas, son conceptos amplios y con perfiles claramente difusos que requieren de ulterior concreción por parte de las administraciones públicas. En esta dirección, la legislación tiene un papel significativo en el desarrollo del gobierno abierto y de las compras abiertas al facilitar el marco en el que las administraciones públicas pueden desarrollar los principios del gobierno abierto y diseñar las diferentes acciones para aplicarlos (Wirtz \& Birkmeyer, 2015, 391).

Para conocer cuál es el reflejo de las compras abiertas en la legislación española es necesario analizar la legislación relativa a la contratación pública y, en particular, al uso de los medios electrónicos, es decir, a la contratación pública electrónica. Sin embargo, no podemos desconocer que las compras abiertas, como en general el gobierno abierto, van más allá del impulso de la administración electrónica. Si esta ha sido más un instrumento de eficacia y eficiencia administrativa, no ha conseguido por el momento tener un impacto significativo en la mejora de la transparencia y la participación ciudadanas en la contratación pública. Por ello, en este análisis también deberemos aproximarnos a las normas que sobre transparencia y gobierno abierto se han ido aprobando en los últimos años en España para ver cuál es su impacto en la contratación pública. 


\subsection{Las compras abiertas en la legislación sobre contratación pública}

La contratación pública electrónica se refiere a la utilización de los medios electrónicos en la contratación pública (Comisión Europea, 2010, 2). La contratación pública electrónica puede mejorar la eficacia y la eficiencia pública pero también puede tener una incidencia positiva en la lucha contra la corrupción. En esta dirección, RoseAckerman afirma que "la tecnología moderna puede ayudar a reducir la corrupción en la contratación" (RoseAckerman \& Truex, 2012, 26).

Los medios electrónicos tienen diversas manifestaciones en la contratación pública extendiéndose a todas las fases de la tramitación del procedimiento de contratación desde la difusión de los anuncios relativos a las licitaciones hasta la adjudicación de los contratos pasando por el suministro del pliego de condiciones o la presentación de las ofertas por los empresarios (Martínez Gutiérrez, 2015).

En la actualidad, el Real Decreto Legislativo 3/2011, de 14 de noviembre, por el que se aprueba el texto refundido de la Ley de Contratos del Sector Público (en adelante, TRLCSP) fomenta el uso de los medios electrónicos en la tramitación de los procedimientos de contratación. En los próximos meses, la legislación española deberá dar un paso adelante mediante el impulso definitivo del uso de los medios electrónicos al trasponer a nuestro ordenamiento lo previsto en la Directiva 2014/24/UE del Parlamento Europeo y del Consejo de 26 de febrero de 2014 sobre contratación pública y por la que se deroga la Directiva 2004/18/CE que reconoce que "los medios de información y comunicación electrónicos pueden simplificar enormemente la publicación de los contratos y aumentar la eficiencia y la transparencia de los procedimientos de contratación. Deben convertirse en el método estándar de comunicación e intercambio de información en los procedimientos de contratación" (considerando 52).

En particular, la legislación vigente prevé diferentes mecanismos en los que se prevé explícitamente el uso de los medios electrónicos en la contratación pública como la publicación en Internet de los pliegos y la documentación complementaria; la creación del perfil del contratante; la adjudicación a través del sistema dinámico de adquisición y de la subasta electrónica; el uso de la notificación electrónica o la formalización del contrato a través de documentos electrónicos.

Como se puede observar, los mecanismos previstos en la legislación española no recogen todas las necesidades que se derivan de los Principios Globales para las Contrataciones Abiertas ni desde la perspectiva de la transparencia ni, mucho menos, desde la perspectiva de la participación, el monitoreo y la supervisión.

Además, no podemos desconocer que, a pesar de los positivos efectos que la contratación electrónica puede tener tanto para la eficacia y la eficiencia de la contratación como para la prevención de la corrupción, "el grado de implantación efectiva de la contratación electrónica es muy inferior a lo esperado inicialmente" (Comisión Europea, 2010, 2). Desde la perspectiva de la aplicación de la legislación sobre contratación pública y del impacto de los principios del gobierno abierto queda todavía un largo camino por recorrer.

\subsection{Las compras abiertas en la legislación sobre transparencia pública}

Como ha sido ampliamente reconocido, cualquier intento de prevenir y luchar contra la corrupción en la contratación pública debe perseguir que esta se gestione en un marco de transparencia (Cerrillo i Martínez, 2012C); (Boix Palop, 2014, 203). En este sentido, Moreno Molina recuerda que "[l]a transparencia es un arma eficaz en la lucha contra la corrupción y los fraudes de todo tipo respecto de los fondos públicos" (Moreno Molina, 2008, 90); (Kaufmann, 2005, 92).

En los últimos meses se han ido aprobando en España diferentes normas relativas a la transparencia pública que regulan la difusión proactiva de información pública, el derecho de acceso a la información pública y la reutilización de la información pública (Guichot Reina, 2014); (Meseguer Yebra, 2015); (Velasco Rico, 2014).

Desde la perspectiva de la contratación pública podemos traer a colación las siguientes cuestiones de interés reguladas en las leyes sobre transparencia y acceso a la información en vigor en España.

En primer lugar, en relación a la difusión proactiva de información, resulta interesante observar la apuesta que han hecho las diferentes normas aprobadas en España por crear portales de transparencia, fomentar la calidad y la accesibilidad de la información y exigir el uso de formatos reutilizables (Cerrillo i Martínez, 2014b); (Cerrillo i Martínez, 2015). Además desde la perspectiva de la información sobre la contratación pública, la legislación sobre transparencia prevé que las administraciones públicas deberán difundir a través de Internet numerosa información relativa a los contratos formalizados, el importe de licitación y de adjudicación, el procedimiento utilizado para su adjudicación, el número de licitadores participantes en el procedimiento y la identidad del adjudicatario. También, se prevé que se difundirá la información relativa a las modificaciones, las decisiones de desistimiento y 
renuncia de los contratos. Asimismo, se deben publicar datos estadísticos relativos a los contratos adjudicados (por todas, véase, el art.7.a Ley 19/2013, de 9 de diciembre). Algunas normas autonómicas han ampliado la información sobre contratación pública que deberán difundir las administraciones públicas, por ejemplo, incluyendo la obligación de difundir más información sobre la adjudicación y la ejecución de los contratos, la subcontratación, etc. (por ejemplo, la Ley catalana 19/2014, de 29 de diciembre, de transparencia, acceso a la información pública y buen gobierno o la Ley 8/2015, de 25 de marzo, de Transparencia de la Actividad Pública y Participación Ciudadana de Aragón).

En segundo lugar, en relación al acceso a la información pública, es evidente que su ejercicio permite conocer toda aquella información que no haya sido difundida de forma proactiva por las propias administraciones públicas. La normativa vigente reconoce el acceso a la información como un derecho subjetivo que puede ejercerse por medios electrónicos. La administración pública tiene el plazo de un mes para resolver las solicitudes y en el caso de que sean denegadas, el solicitante puede interponer una reclamación ante el Consejo de Transparencia y Buen Gobierno u órgano o entidad autonómica análoga. No obstante, respecto al acceso a la información deben tenerse presentes los límites previstos en la Ley 19/2013 entre los que destacan, por su especial incidencia en la contratación pública, los relativos al secreto profesional y la protección de la propiedad intelectual e industrial (art.14 Ley 19/2013). Asimismo, no se puede desconocer que el alcance de los límites al derecho de acceso puede verse ampliado por la propia voluntad del licitador puesto que la legislación sobre contratación pública prevé que "los órganos de contratación no podrán divulgar la información facilitada por los empresarios que éstos hayan designado como confidencial; este carácter afecta, en particular, a los secretos técnicos o comerciales y a los aspectos confidenciales de las ofertas" (art.140 TRLCSP).

Finalmente, en tercer lugar, respecto a la reutilización de la información pública, las normas sobre transparencia poco añaden a lo que ya regulaba la Ley 37/2007, de 16 de noviembre, de reutilización de la información pública que determina las condiciones y los procedimientos para que se pueda reutilizar la información pública. En particular, la legislación sobre transparencia básicamente insiste sobre la necesidad de difundir la información en formatos que permitan su reutilización (art.11.c Ley 19/2013). Más allá, la legislación española no recoge explícitamente todos los principios de datos abiertos aunque su contenido encuentra perfecto acomodo tanto en los principios de la administración electrónica como en la normativa básica sobre transparencia, acceso y reutilización de la información pública en particular después de la aprobación de la modificación de la Ley 37/2007, de 16 de noviembre por la Ley 18/2015, de 9 de julio (Cerrillo i Martínez, 2014d).

La legislación sobre transparencia incorpora buena parte de las obligaciones que se derivan de los Principios Globales para las Contrataciones Abiertas. Sin embargo, un análisis de la legislación a la vista de los Principios Globales para las Contrataciones Abiertas permite observar como la Ley 19/2013, ni en su mayoría las normas autonómicas de desarrollo, no obliga a difundir información sobre los estudios previos, sobre los criterios de evaluación, sobre la capacidad de los licitadores ni los conflictos de intereses en que eventualmente puedan encontrarse, sobre la evaluación de la ejecución de los contratos.

\subsection{Las compras abiertas en la legislación sobre gobierno abierto}

El gobierno abierto es absolutamente esencial para prevenir y luchar contra la corrupción (Boix Palop, 2014).

Más allá de la regulación de la transparencia a la que hemos hecho referencia en el epígrafe anterior, diversas normas prevén mecanismos a través de los que se canalizan los principios de de participación y colaboración.

En efecto, en los últimos años diversas Comunidades Autónomas han adoptado normas relativas al gobierno abierto. Una de ellas es la Ley catalana 19/2014, de 29 de diciembre, de transparencia, acceso a la información pública y buen gobierno que, como destaca su preámbulo, caracteriza el gobierno abierto como "un sistema de actuación y gestión de los asuntos públicos que hace posible un diálogo permanente entre la Administración y los ciudadanos, su participación y colaboración en la definición de las políticas públicas y una mejor transparencia en la rendición de cuentas y la exigencia de responsabilidades". Asimismo, se observa como el gobierno abierto aprovecha "las ventajas de los medios electrónicos para configurar un sistema de relaciones entre las administraciones y la ciudadanía más interactivo y más participativo".

La Ley 19/2024 define los principios en que se basa el gobierno abierto, establece diversas medidas para fomentar el gobierno abierto y para garantizar su efectividad y regula diversos instrumentos de buen gobierno. Entre ellos destaca la intervención de la ciudadanía, de manera individual o colectiva, por medio de entidades de carácter representativo, en la definición y la aplicación de las políticas públicas y el fomento de la participación y la colaboración ciudadanas en el seguimiento y la evaluación de la aplicación de las decisiones por parte de las administraciones públicas. 
Asimismo concreta algunos de los mecanismos a través de los que se manifiestan los principios del gobierno abierto donde destacan particularmente los relativos a la transparencia y en menor medida, por su remisión a otras normas, los relativos a la participación y la colaboración ciudadanas.

Más allá de estos mecanismos genéricos que prevé la ley catalana y que también se pueden encontrar en otras normas autonómicas (Ley 4/2013, de 21 de mayo, de Gobierno Abierto de Extremadura o la Ley 8/2015, de 25 de marzo, de Transparencia de la Actividad Pública y Participación Ciudadana de Aragón), la legislación española no regula explícitamente mecanismos de participación y colaboración ciudadana en el ámbito de la contratación pública.

A pesar de las normas aprobadas, aún queda un largo camino por recorrer desde la perspectiva de la regulación del gobierno abierto en España. Las normas existentes no facilitan directamente el monitoreo y la supervisión de la actividad contractual como tampoco la posibilidad de que la ciudanía retroalimente a los órganos de contratación pública sobre su desempeño de la actividad contractual ni la participación ciudadana durante la ejecución de los contratos públicos aspectos todos ellos previstos en los Principios Globales para las Contrataciones Abiertas.

\section{COMPRAS ABIERTAS Y LUCHA CONTRA LA CORRUPCIÓN}

El gobierno abierto no sólo permite fortalecer las relaciones entre las administraciones públicas y la ciudadanía sino que también puede facilitar la prevención y la lucha contra la corrupción.

En el marco de la regulación sobre la contratación pública electrónica, la transparencia pública y el gobierno abierto que hemos expuesto en el epígrafe anterior, existe la posibilidad de desarrollar diferentes proyectos que permitan fomentar los principios del gobierno abierto en la contratación pública y, en última instancia, facilitar la prevención y la lucha contra la corrupción a través de una mayor implicación ciudadana.

En las próximas páginas nos proponemos valorar cómo el gobierno abierto y, en particular, las compras abiertas, pueden contribuir a la prevención y la lucha contra la corrupción en la contratación pública. Para ello, nos aproximaremos en primer lugar a las principales manifestaciones de la corrupción en las diferentes fases de la contratación pública para, a continuación, analizar qué alcance se debe dar a las compras abiertas para, más allá de facilitar la eficacia y la eficiencia de la contratación pública, contribuir a la prevención y la lucha contra las diferentes manifestaciones de la corrupción en la contratación pública.

\subsection{Las principales manifestaciones de la corrupción en la contratación pública}

Como punto de partida, podemos recordar que la corrupción es aquella situación en la que un cargo o empleado público aprovecha un conflicto de intereses para la satifacción de un interés propio que se concreta en la obtención de una ganancia incumpliendo el marco de referencia existente (Cerrillo i Martínez, 2014a, 40). La corrupción supone un abuso del poder que le ha sido confiado a un determinado agente público para obtener ganancias privadas (OECD, 2005, 32).

Son muchas las situaciones que se pueden dar en la contratación pública que enmascaran un conflicto de interés o un caso de corrupción. Sin embargo, no todas las irregularidades que se pueden dar en una licitación pública o en la ejecución de un contrato público deben juzgarse como un caso de corrupción puesto que debe existir un uso desviado del poder público por parte de un cargo o empleado público en beneficio propio o personal para poder llegar a tal conclusión (Cerrillo i Martínez, 2014a, 47).

En la formación de la demanda pública de un contrato, existen diversos comportamientos o situaciones que pueden vincularse a casos de corrupción como cuando los contratos públicos se celebran en interés del funcionario público o en interés de terceros fruto de la demanda inducida por los empresarios. En esta fase también se pueden incluir aquellos acuerdos o colusiones entre diferentes empresarios que en determinados casos entrañan a su vez casos de corrupción.

Durante la adjudicación del contrato también se observan actuaciones que pueden resultar de posibles prácticas corruptas como son la contratación directa; la inexistencia de contrato o contratación indebida; el fraccionamiento de los contratos públicos; la variación en el curso del contrato a través de la modificación unilateral de los contratos (Martín Rebollo, 2009, 42); o la utilización arbitraria de las prórrogas (Castro Cuenca, 2009, 22). Además, se debe añadir el soborno para la adjudicación del contrato público o la adjudicación como resultado de influencias indebidas que puedan limitar los requisitos para participar en una licitación (Jareño Leal, 2011).

Finalmente, en la ejecución del contrato, los sobrecostes de las obras; el abuso de la subcontratación; la revisión de precios en relación a las ofertas anormalmente bajas; o en la expedición de facturas y documentos falsos pueden ser también manifestaciones de casos de corrupción. 


\subsection{El impacto de las compras abiertas en la prevención y la lucha contra la corrupción}

Cada una de las situaciones que se han apuntado en el epígrafe anterior genera unos datos y unas informaciones que, con frecuencia son desconocidas tanto por las administraciones públicas como por la ciudadanía. Estas situaciones también implican a diferentes actores. Nos encontramos ante situaciones muy diversas que, generalmente, se caracterizan además de perseguir el beneficio propio o de tercero abusando de las potestades públicas de las que se es titular, por el hecho de que se dan en entornos caracterizados por la opacidad y el secreto (OECD, 2009, 10) así como por la informalidad (Dreher \& Schneider, 2010) que posteriormente logra ver la luz pública y adoptar las formas previstas siguiendo los cauces previstos por la legislación. De este modo, a no ser que se tenga un acceso a esos entornos será necesario bien limitarlos al máximo bien identificar evidencias sobre su existencia indirectamente.

El conocimiento de la información vinculada a un caso de corrupción que permita identificar situaciones potenciales de corrupción y la participación y colaboración de todos aquellos que puedan tener conocimiento bien de la información bien de las situaciones de corrupción es el principal reto que se deriva de la aplicación de los principios de gobierno abierto en la contratación pública.

Por ello, podemos partir de la idea que la transparencia que se deriva de la extensión del gobierno abierto incide positivamente en dificultar buena parte de las situaciones de corrupción a que se ha hecho referencia y, en el caso de que surjan, poderlas detectar y hacerles frente (Boix Palop, 2014, 205).

En esta dirección, las compras abiertas y, en particular, los datos abiertos sobre contratación pública pueden generar numerosos beneficios como estrategia anticorrupción (Beke \& Blomeyer\&Sanz, 2015, 31).

En primer lugar, aportando luz a estos entornos donde se adoptan los acuerdos o se toman las decisiones informales que dan lugar a casos de corrupción que permite prevenir su existencia y, en el caso de que se produzcan, identificarlos y luchar contra ellos. El incremento de la información y los datos sobre los contratos públicos en formatos que faciliten la reutilización constituye un potente mecanismo que unido al uso de metodologías propias del Big Data permitirá conocer mejor como se desarrolla la actividad contractual y, eventualmente identificar situaciones irregulares, comportamientos y conductas ilegales y casos de corrupción a partir del análisis cruzado de ingentes cantidades de datos relacionados con la contratación pública.

En segundo lugar, facilitando la implicación ciudadana en la prevención y la lucha contra la corrupción. Gracias a la tecnología, los ciudadanos pueden participar en el monitoreo, seguimiento y supervisión de la actividad contractual. También pueden colaboran en la denuncia o alerta de casos de corrupción preservando su identidad o garantizando su seguridad.

\subsection{Los datos abiertos y la prevención eficaz de la corrupción}

Como recuerda Tschorne, “[c]ontar con información de calidad y en forma oportuna permite hacer más eficiente y eficaz la toma de decisiones en los procesos de planificación, diseño, construcción, explotación y fiscalización de las obras públicas; disminuir los riesgos financieros y de operación en la prestación de los servicios y dar cuenta a las autoridades y a la ciudadanía en general de la gestión de las obras y de los servicios comprometidos" (Tschorne Berestesky, 2012, 166).

Para ello, es necesario avanzar en la difusión de datos abiertos que permitan su reutilización y análisis. Como recuerda Tim Berners-Lee, "los datos de las administraciones públicas se están poniendo en línea para aumentar la rendición de cuentas, facilitar información útil sobre el mundo, y para que las administraciones públicas del país y el mundo funcionen de manera más eficiente" (Berners-Lee, 2009).

Los datos abiertos son aquellos datos que las administraciones públicas ponen a disposición de la ciudadanía para que puedan ser fácilmente reutilizados gracias a que son fáciles de localizar, de reutilizar y no están sometidos a restricciones jurídicas que limiten o dificulten su reutilización (propiedad intelectual, datos personales, etc.) (Cerrillo i Martínez, 2014d).

La reutilización de los datos abiertos persigue, principalmente, la producción de nuevas informaciones y a la provisión de servicios basados en ellos. Sin embargo, no se puede ignorar su impacto en la mejora de la transparencia (Cerrillo i Martínez, 2012a) ni en la reducción de los niveles de corrupción (Granickas, 2014, 4) gracias a la implicación ciudadana en el análisis y supervisión de la actividad pública a través de la reutilización de los datos públicos y su ulterior difusión a través de formatos fácilmente comprensibles por la ciudadanía.

Para que los datos abiertos puedan incidir positivamente en la prevención y la lucha contra la corrupción es necesario que se den dos circunstancias. En primer lugar, que los datos que se difundan sean suficientes. En segundo lugar, que los datos se difundan en formatos que faciliten su reutilización. 


\section{a) La difusión de suficientes datos abiertos sobre contratación pública}

Por lo que se refiere a la suficiencia de los datos ya hemos avanzado que en la actualidad las leyes sobre transparencia que están en vigor en España obligan a las administraciones públicas a difundir amplios conjuntos de datos en materia de contratación pública. Sin embargo, también hemos podido observar como la mayoría de estos datos están vinculados a la fase de adjudicación del contrato y en menor medida tanto a la preparación previa como a la ejecución posterior. Precisamente tanto la preparación como la ejecución son momentos en los que se pueden dar diferentes situaciones que pueden encubrir abusos y casos de corrupción como hemos indicado anteriormente (demanda inducida, abuso de la subcontratación o de las modificaciones contractuales, etc.).

Por ello, es necesario que más allá de lo que prevé la legislación vigente tanto en materia de contratación pública como en materia de transparencia pública puedan ampliarse por la vía de la práctica los conjuntos de datos que difunden las administraciones públicas relativos a estas otras fases de la contratación pública en la línea de lo que prevén los Principios Globales para las Contrataciones Abiertas. Como apunta Sanmartín "[l]a información sobre aspectos como las desviaciones en los precios y en los plazos de ejecución, la aprobación de contratos complementarios o el grado de subcontratación, es imprescindible para hacer un juicio sobre la correcta gestión de la contratación pública" (Sanmartín Mora, 2014).

En el pasado ya sucedió cuando antes de que así lo previese la legislación sobre transparencia pública, algunas administraciones públicas daban cuenta en Internet de la información relativa a los contratos menores o a los estudios e informes adjudicados.

\section{b) La difusión de datos sobre contratación pública en formatos abiertos}

Por lo que respecta a la difusión de los datos en formatos que faciliten su reutilización, debemos partir de la idea de que los datos deben ser abiertos. Se consideran abiertos cuando se difunden cumpliendo los conocidos principios de datos abiertos propuestos por la Sunlight Foundation: completos, primarios, oportunos, accesibles, procesables electrónicamente, no discriminatorios, uso de estándares abiertos en la difusión de la información, sin licencia, permanencia y sin costes (Cerrillo i Martínez, 2014d, 64).

Estos principios se han visto concretados en el ámbito de las compras abiertas. Al respecto, podemos traer a colación los estándares de datos para las contrataciones abiertas desarrollados por la Alianza para las Contrataciones Abiertas con la colaboración de la Fundación World Wide Web y el Banco Mundial. El objetivo de estos estándares es promover la divulgación de los datos sobre la contratación pública y, por ende, mejorar la eficiencia pública y luchar contra la corrupción. Los estándares persiguen que las administraciones públicas difundan los datos sobre contratación pública con formatos estandarizados y de manera ágil y que su reutilización sea fácil y barata y que permita la comparación. Si no se cumplen estas características que persiguen la calidad de los datos, es imposible el monitoreo y la evaluación de la actividad pública (United Nations, 2014).

El uso de los estándares de Datos para las Contrataciones Abiertas puede facilitar la obtención de una mayor rentabilidad en las contrataciones; la detección de fraudes y casos de corrupción; una mayor competencia por contratos públicos y la supervisión de la prestación de servicios.

La Sunlight Foundation también ha elaborado los Procurement Open Data Guidelines que recogen los principios que deben caracterizar la difusión de los datos relativos a la contratación pública5:

\section{El Proceso de Contratación}

1. Todas las leyes y políticas relativas a la contratación pública deben ser accesibles al público.

2. Las licitaciones deben estar a disposición del público y debe permanecer incluso cuando haya finalizado el plazo establecido.

3. Los datos relativos a la contratación directa u otros contratos que no requieren licitación pública, también deben ser publicados.

4. Los documentos de cada contrato, así como información de contacto de todos los licitadores, debe darse a conocer públicamente.

5. Las comunicaciones entre los empresarios y los empleados públicos durante el proceso de licitación debe llevarse a cabo en un foro público.

5 http://sunlightfoundation.com/procurement/opendataguidelines (última consulta: julio 2015). 
6. Las adjudicaciones deben difundirse públicamente tan pronto como se adjudiquen, incluidas las motivaciones y los criterios para la adjudicación.

7. El texto completo de cualquier contrato adjudicado, así como cualquier modificación debe ser publicado proactivamente en línea facilitando una información mínima como el adjudicatario, el importe, la fecha, etc., como datos estructurados.

8. La información relativa a los procedimientos de resolución de conflictos debe estar disponible en todas las fases del procedimiento de contratación.

Los participantes en el Proceso de Contratación

9. Las bases de datos que contienen información actual e histórica sobre la ejecución de los contratos debe estar disponible públicamente.

10. La información relativa al estado de los contratos debe estar a disposición del público en todo momento y más allá de la finalización del contrato.

11. Si una parte importante de un contrato es subcontratada, los datos disponibles para los contratistas también debe estar disponible para el subcontratista.

12. Debe haber un identificador único para los contratos que se utiliza constantemente el proceso de contratación.

13. Las entidades adjudicatarias deben ser identificadas por un identificador único, no-propietario, a disposición del público.

Cómo liberar a esta información

14. Cuando sea posible, los gobiernos deben tratar de adoptar las normas existentes para la liberación de los datos de contratación para fomentar la interoperabilidad interfronteriza de los datos.

15. Todos los datos mencionados anteriormente deben estar disponibles como datos estructurados en formatos legibles por una máquina. También debería estar disponible en línea y a granel.

16. Todos los datos mencionados anteriormente deben ser accesibles de forma gratuita y no deben requerir registro, ni en línea ni en persona.

17. Todos los datos deben publicarse en el momento oportuno, y de forma continua durante todo el año fiscal. La divulgación retardada para proteger la confidencialidad de la información debe ser excepcional.

\section{CUADRO 2: PROCUREMENT OPEN DATA GUIDELINES}

Como hemos tenido ocasión de señalar anteriormente, la legislación española recoge directa o indirectamente algunos de los principios que caracterizan los datos abiertos. De todos modos, otros no están explícitamente previstos ni en la legislación sobre contratación pública ni en la relativa a la transparencia pública y el gobierno abierto. En particular, no se prevé durante cuánto tiempo debe difundirse la información (principio 3); la obligación de que las comunicaciones entre empresarios y órganos de contratación se lleve a cabo en foros públicos (principio 5); el plazo para la difusión de la información relativa a las adjudicaciones (principio 6); la obligación de publicar el texto completo del contrato (principio 7); la publicación de bases de datos con información actual e histórica sobre la ejecución (principio 9); la existencia de un identificador único para los contratos y los adjudicatarios (principios 12 y 13); la interoperabilidad (principio 14); la obligación de publicar todos los datos como datos estructurados en formatos legibles por una máquina, en línea y a granel (principio 15); de forma oportuna y continua (principio 17).

Más allá de la no regulación de estos aspectos, la práctica de la mayoría de las administraciones públicas españolas dista mucho de garantizar la aplicación de estos principios en la difusión de la información sobre contratos públicos a través de Internet por lo que las posibilidades de llegar a conclusiones de valor se verán muy limitadas.

\section{c) ¿Qué hacer con los datos abiertos sobre contratación pública?}

Tan importante a los efectos que estamos tratando es que las administraciones públicas difundan los datos y la información requerida en los formatos establecidos como que la ciudadanía analice, interprete y extraiga conclusiones de estos datos. 
Según la Association of Certified Fraud Examiners (ACFE), casi el 50\% de los casos de fraude y corrupción son detectados por delación o por accidente mientras que únicamente el $14 \%$ lo son fruto de una auditoría interna (Ernst \& Young, 2012, 2). Por ello, son necesarias nuevas metodologías que a través del análisis de datos puedan detectar situaciones irregulares que puedan esconder casos de corrupción.

Por lo tanto, el reto no está sólo en difundir la información con la calidad y reusabilidad necesaria sino particularmente en poder analizar dicha información y extraer conclusiones que permitan prevenir y luchar contra la corrupción en la contratación pública a través del seguimiento y el monitoreo de todas las fases de los procedimientos de contratación pública.

Los datos abiertos relativos a la contratación pública pueden ser tan numerosos que pueden dar lugar a conjuntos extremadamente grandes y complejos cuyo análisis a través de las metodologías del Big Data permite llegar a conclusiones imposibles de alcanzar previamente con otras metodologías (Bertot, Gorham, Jaeger, Sarin, \& Choi, 2014).

En efecto, la gran cantidad de datos que están estrechamente vinculados a la contratación pública, exige utilizar metodologías de análisis que permitan extraer conclusiones útiles para supervisar y monitorizar la contratación pública. En esta dirección, Ernst \& Young destacaba recientemente que "AntiCorruption Compliance Now Requires Big Data Analytics", "Anti-bribery and corruption analytics test of controls incorporate big data concepts that integrate multiple data sources: third-party watch lists; transactional data; text mining; and even social media and email to prioritize and isolate areas of risk or rogue activity (EY Center for Board Matters, 2014, 7).

Con la expresión Big Data suele designarse una metodología que se ha desarrollado en los últimos años para analizar grandes cantidades de datos para identificar correlaciones entre ellos. Con esta metodología se persigue extraer conclusiones del uso de la gran cantidad de datos que deriva de la creciente capacidad de almacenamiento o de los sistemas para cruzar datos. Ilustraba esta idea hace unos años Stephen Baker en su conocido libro Numerati al afirmar que "[n]os están vigilando. Una llamada con el móvil, un pago con tarjeta de crédito, un clic en internet... y cada uno de nuestros pasos queda registrado en monumentales bases de datos. Toda esta información resulta insignificante por separado, pero agrupada revela incluso nuestros secretos más inconfesables" (Baker, 2009).

Gracias al Big Data se pueden generar perfiles de contratantes y licitadores así como de procedimientos de licitación que se pueden segregar en función de la cantidad de la licitación, del procedimiento utilizado, etc. que pueden contribuir en la lucha contra la corrupción al identificar pautas de comportamiento, conductas o situaciones que presenten ciertas irregularidades que pueden eventualmente esconder un caso de corrupción. Un aspecto que puede resultar de utilidad es el de utilizar identificadores únicos tanto para los contratos como para los licitadores. De este modo, se facilita tanto su seguimiento como su comparación.

El Big Data facilita el análisis estadístico que permita identificar tendencias o potenciales anomalías, la visualización gráfica de datos para identificar áreas de riesgo, el análisis del texto de correos electrónicos u otras comunicaciones para detectar relaciones irregulares que puedan esconder casos de corrupción.

Ya existen algunos proyectos relativos al uso del Big Data en la lucha contra la corrupción fuera de España. Un ejemplo, lo encontramos en Georgia donde Transparencia Internacional ha impulsado un portal de código abierto para monitorizar y analizar la contratación pública que extrae datos de los portales de contratación pública y la presenta en formatos comprensibles para la ciudadanía que a su vez pueden generar perfiles de los procedimientos de contratación realizados por la Administración pública o perfiles de las empresas licitadoras ${ }^{6}$.

\subsection{Mecanismos para la participación y la colaboración ciudadana en la lucha contra la corrupción}

La ciudanía puede no solo acceder a los datos que se difunden sobre la contratación pública sino que puede participar en su análisis con la finalidad de supervisar y monitorizar la actividad contractual de las administraciones públicas. Esta participación ciudadana puede perseguir tener un mejor conocimiento de la actividad contractual, por ejemplo, por parte de empresas licitadoras para poder presentar mejores propuestas, conocer mejor a los competidores o a la propia administración pública. Pero también puede perseguir identificar situaciones que escondan irregularidades, conflictos de intereses o casos de corrupción.

La participación ciudadana en el análisis de los datos sobre contratación pública puede llevarse a cabo de manera individual, organizada o mediada, por ejemplo, a través de la prensa y el periodismo de datos. Incluso en

6 http://sunlightfoundation.com/blog/2014/01/16/opengov-voices-how-georgia-is-handling-procurement-transparency/ (última consulta: julio 2015). 
ocasiones se organizan eventos que tienen por objetivo analizar conjuntos de datos con la finalidad de encontrar correlaciones que puedan ser de interés (hackathons) 7 .

En España aún son pocas las iniciativas ciudadanas en esta dirección pero en los últimos años ya han surgido algunas. Por ejemplo, la Fundación ciudadana Civio ha impulsado el proyecto ¿Quién cobra la obra? Este proyecto persigue crear una base de datos pública y reutilizable sobre los contratos públicos a partir de la información que se difunde en Boletín Oficial del Estado que incluya los datos más relevantes (precio, fecha de ejecución, administración pública que paga la obra, adjudicataria) a partir de la cual se puedan hacer todo tipo de visualizaciones y análisis sobre cómo funciona la contratación pública en Españå ${ }^{8}$.

Nuevamente en este caso, la tecnología facilita instrumentos para la participación ciudadana en la lucha contra la corrupción como instrumento para el análisis de los datos pero también para la difusión de los resultados alcanzados (Davies \& Fumega, 2014, 19).

A la vista de los resultados observados, los ciudadanos también pueden colaborar en la lucha contra la corrupción denunciando o informando a las autoridades competentes sobre las situaciones identificadas. En los últimos años desde diferentes instituciones internacionales se ha llamado la atención sobre la necesidad de impulsar mecanismos que faciliten la información o denuncia de casos de corrupción ${ }^{9}$. Por ejemplo, Transparencia Internacional en su resolución de octubre de 2009 sobre la protección del denunciante (whistleblower) apunta que "la denuncia puede ser un instrumento crucial para detectar e informar sobre la corrupción, el fraude y la mala gestión en el sector público, el privado y el no lucrativo" ${ }^{10}$. Los medios electrónicos pueden contribuir tanto a facilitar la información o la denuncia de casos de corrupción que haya podido identificar la ciudadanía como a proteger su identidad o garantizar su seguridad frente a posibles represalias (Cerrillo i Martínez, 2014C).

En esta dirección, organismos como la Oficina Europea de Lucha contra el Fraude han desarrollado mecanismos de denuncia en línea (Fraud Notification System) en los que el denunciante puede informar sobre el tipo de fraude identificado, el momento en el que ocurrió así como toda aquella información que facilite la investigación y donde se garantiza la protección de su identidad a través de la creación de una cuenta de correo segura en la que de forma anónima el personal de dicha oficina puede ponerse en contacto con el denunciante ${ }^{11}$. En otros países, se han creado canales específicos para la denuncia de las irregularidades detectadas en la contratación pública ${ }^{12}$.

\section{REFLEXIONES FINALES: HACIA UNA FUTURA REGULACIÓN DE LAS COMPRAS ABIERTAS EN ESPAÑA QUE FACILITE LA PREVENCIÓN DE LA CORRUPCIÓN}

Las compras abiertas tienen aplicaciones de gran interés en la contratación pública que pueden contribuir eficazmente a la prevención y la lucha contra la corrupción a través de la difusión de datos abiertos sobre contratación pública y su utilización por la ciudadanía para supervisar y monitorizar la contratación pública y, en su caso, informar o denunciar, ante las autoridades competentes para su investigación y sanción, las situaciones irregulares que se hayan podido identificar que escondan conflictos de intereses o casos de corrupción. Para que los datos abiertos sobre la contratación pública y su tratamiento a través de las metodologías del Big Data pueden ser una herramienta útil para la prevención y la lucha contra la corrupción, es necesario que sean suficientes, de calidad, en formatos reutilizables y comparables.

Como hemos tenido oportunidad de analizar en las páginas anteriores las compras abiertas consisten en el uso de la tecnología por las administraciones públicas y en la extensión de la transparencia, la participación y la colaboración ciudadanas en su tramitación y seguimiento. Pero, más allá las compras abiertas implican un cambio de valores (Calderón \& Lorenzo, 2010) y, en particular, un impulso de la innovación en las administraciones públicas y entre la ciudadanía. Este cambio de valores debe ir acompañado de un cambio en la cultura administrativa y cívica y del impulso de nuevos instrumentos de relación, participación y colaboración que exigirá un liderazgo político decidido. Todo ello demandará la formación de todos los actores implicados (Chêne, 2012, 4).

En la actualidad la legislación española recoge algunos de los principios y mecanismos de las compras abier-

7 Véase, como ejemplo, http://www.ledevoir.com/societe/actualites-en-societe/363757/hackathon-contre-la-corruption (última consulta: julio 2015).

8 http://www.civio.es/2014/10/quien-cobra-la-obra-ayudanos-a-investigar-la-contratacion-de-obra-civil/ (última consulta: julio 2015).

9 Resulta de interés el proyecto Digital Whistleblower (Digiwhist) que persigue facilitar a la ciudadanía herramientas para incrementar la transparencia pública y la rendición de cuentas. Véase más información en: https://www.hertie-school.org/facultyandresearch/researchprojects/research-projects/digiwhist/ (última consulta: julio de 2015).

10 Accesible en: http://www.transparency.org/files/content/activity/2009_PrinciplesForWhistleblowingLegislation_EN.pdf (última consulta: julio de 2015).

11 Accesible en: https://fns.olaf.europa.eu/cgi-bin/questionnaire_cgi (última consulta: julio de 2015).

12 Véase, por ejemplo, http://quejas.funcionpublica.gob.mx/PortalQD-web/xhtml/queja/capturarQueja.jsf (última consulta: julio de 2015). 
tas. Sin embargo, hemos tenido oportunidad de señalar que aún queda un largo camino por recorrer para garantizar jurídicamente las compras abiertas en toda su extensión en España. Por ello, por el momento su pleno desarrollo se debe llevar a cabo por iniciativa de cada administración pública en el marco de su compromiso con el gobierno abierto y con la lucha contra la corrupción.

Ante las limitaciones observadas de la legislación vigente, sería de desear que la futura ley de contratación pública por la que se transponga al ordenamiento jurídico español la Directiva 2014/24/UE del Parlamento Europeo y del Consejo de 26 de febrero de 2014 sobre contratación pública y por la que se deroga la Directiva 2004/18/CE incorporase decididamente los principios de las compras abiertas analizados en las páginas anteriores.

En este sentido, más allá de potenciar el uso de la tecnología en la contratación pública como ha sido ya propuesto (Martínez Gutiérrez, 2015, en particular, 254 y ss.), la futura ley de contratación pública también debería reconocer explícitamente los principios de las compras abiertas y garantizar su aplicación a través de diferentes mecanismos.

En primer lugar, estableciendo la obligación de difundir en formato de datos abiertos los datos que actualmente ya se difunden a través del perfil del contratante y de los portales de transparencia y páginas web de las administraciones públicas y de otros poderes adjudicadores.

En segundo lugar, ampliando el conjunto de datos a difundir más allá de lo actualmente previsto tanto en la legislación sobre contratos públicos como en la legislación sobre transparencia hasta recoger no sólo los datos incluidos en los Principios Globales para las Contrataciones Abiertas sino todos aquellos vinculados a las situaciones que con frecuencia encubren casos de corrupción (por ejemplo, estudios previos, subcontratación, etc.).

En tercer lugar, facilitando la información y la denuncia ciudadana de aquellas situaciones irregulares, conflictos de intereses y casos de corrupción que se puedan detectar a la vista del análisis y el monitoreo de dichos datos mediante la creación de agencias de integridad en materia de contratación pública y el establecimiento de mecanismos electrónicos para la información y la denuncia o, en su caso, la atribución de esta función a órganos actualmente ya en funcionamiento con competencias en materia de contratación pública.

En cuarto lugar, ampliando el ámbito subjetivo legitimado para interponer el recurso especial en materia de contratación para reconocer una acción pública respecto a la contratación pública a todos aquellas personas que tengan un conocimiento efectivo de irregularidades vinculadas a conflictos de intereses y de corrupción.

\section{REFERENCIAS BIBLIOGRÁFICAS}

Baker, S. (2009). Numerati: Saben todo de ti. Barcelona: Seix Barral.

Beke, M. B., \& Blomeyer\&Sanz. (2015). Towards a European Strategy to Reduce Corruption by Enhancing the Use of Open Data. National Research Spain.

Berners-Lee, T. (2009). Putting Government data online.

Bertot, J. C., Gorham, U., Jaeger, P. T., Sarin, L. C., \& Choi, H. (2014). "Big data, open government and e-government: Issues, policies and recommendations”. Information Polity, 19(1), 5-16. DOI: 10.3233/IP-140328.

Bertot, J. C., Jaeger, P. T., \& Grimes, J. M. (2010). “Using ICTs to create a culture of transparency: E-government and social media as openness and anti-corruption tools for societies". Government Information Quarterly, 27(3), 264-271. doi: http://dx.doi.org/10.1016/j.giq.2010.03.001.

Boix Palop, A. (2014). “Open Government y lucha contra la corrupción”. En AAVV (Ed.), La era de la \#Políticazptoo.

Calderón, C., \& Lorenzo, S. (Eds.). (2010). Open Government: Gobierno Abierto. Alcalá la Real: Algón.

Castro Cuenca, C. G. (2009). La corrupción en la contratación pública en Europa. Salamanca: Ratio Legis.

Cerrillo i Martínez, A. (2012a). "Fundamental interest and open data for re-use". International Journal of Law and Information, 20(3), 203-222. DOI: 10.1093/ijlit/eas014.

Cerrillo i Martínez, A. (2012b). "The re-use of public sector information in Europe and its impact on transparency". European Law Journal, 18(6), 770-792.

Cerrillo i Martínez, A. (2012c). "Transparencia administrativa y lucha contra la corrupción en la Administración local”. Anuario del Gobierno Local 2011, 277-313.

Cerrillo i Martínez, A. (2014a). El principio de integridad en la contratación pública. Mecanismos para la prevención de los conflictos de intereses y la lucha contra la corrupción. Cizur Menor: Aranzadi-Thomson-Reuters.

Cerrillo i Martínez, A. (2014b). “El uso de medios electrónicos en la difusión de información pública”. En M. Fernández Salmerón \& J. Valero Torrijos (Eds.), Régimen jurídico de la transparencia en el sector público: acceso, uso y reutilización de la información administrativa. Cizur Menor: Thomson-Aranzadi.

Cerrillo i Martínez, A. (2014C). "La colaboración ciudadana en la lucha contra la corrupción a través de medios electrónicos”. Revista Aranzadi de Derecho y Nuevas Tecnologías, 35, 43-71. 
Cerrillo i Martínez, A. (2014d). “Los principios de datos abiertos en la legislación española”. Revista de Internet, Derecho y Política, 19, 62-77.

Cerrillo i Martínez, A. (2015). “La transparència pública”. En A. Cerrillo i Martínez \& J. Ponce Solé (Eds.), Transparència, accés a la informació i bon govern a Catalunya. Comentaris de la Llei 19/2014, de 9 de desembre. Barcelona: Editorial UOC-Escola d'Administració Pública de Catalunya.

Chêne, M. (2012). The benefits of open contracting: Anticorruption Resource Centre-Transparency International.

Chun, S. A. S., Stuart; Sandoval, Rodrigo; Hovy, Eduard. (2010). "Government 2.0: Making connection between citizens, data and government". Information Polity, 15.

Comisión Europea. (2010). Libro verde sobre la generalización del recurso a la contratación pública electrónica en la UE-COM (2010) 571 final.

Corojan, A., \& Campos, E. (2011). "Gobierno abierto: alcance e implicaciones". Documentos de trabajo. Fundación ideas, $03 / 2011$.

Cruz-Rubio, C. N. (2014). Hacia el gobierno abierto: una caja de herramientas. Washington: Organización de los Estados Americanos.

Dassen, N., \& Veyra, J. C. (Eds.). (2012). Gobierno Abierto y Transparencia Focalizada. Tendencias y desafíos para América Latina y el Caribe. Washington, D.C.: Banco Interamericano de Desarrollo.

Davies, T., \& Fumega, S. (2014). Mixed incentives: Adopting ICT innovations for transparency, accountability, and anti-corruption: AntiCorruption Resource Centre.

Dreher, A., \& Schneider, F. (2010). "Corruption and the shadow economy: an empirical analysis". Public Choice, 144(1-2), 215-238. DOI: 10.1007/s11127-009-9513-0.

Ernst \& Young. (2012). Anti-bribery and corruption analytics. Integrating anti-fraud analytics into your anti-bribery and corruption compliance approach.

European Commission. (2014a). Businesses' attitudes towards corruption in the EU.

European Commission. (2014b). EU Anti-corruption Report COM (2014) 38 final.

EY Center for Board Matters. (2014). "The bar is raised. Anti-corruption compliance now requires big data analytics". Board Matters Quarterly.

Granickas, K. (2014). “Open Data as a Tool to Fight Corruption”. European Public Sector Information Platform Topic Report, 2014/04.

Guichot Reina, E. (Ed.). (2014). Transparencia, Acceso a la Información y Buen Gobierno. Estudio de la Ley 19/2013, de 9 de diciembre. Madrid: Tecnos.

Jareño Leal, Á. (2011). Corrupción y delincuencia de los funcionarios en la contratación pública. Madrid: lustel.

Kaufmann, D. (2005). "Myths and Realities of Governance and Corruption”. Working Paper Series. The Brookings Institution. DOI: 10.2139/ssrn.829244.

Lee, G., \& Kwak, Y. H. (2012). “An Open Government Maturity Model for Social Media-based Public Engagement”. Government Information Quarterly, 29, 492-503. DOI: 10.1016/j.giq.2012.06.001.

Marchessault, L. (2013). Open Contracting Principles reflect norms and best practices from around the world.

Martín Rebollo, L. (2009). "La modificación de los contratos: régimen, regulación y consecuencias de una práctica generalizada que supone un riesgo al principio licitatorio y a la idea de transparencia". Revista española de la función consultiva, 12, 41-106.

Martínez Gutiérrez, R. (2015). La contratación pública electrónica. Análisis y propuestas de transposición de las Directivas Comunitarias de 2014. València: Tirant lo Blanch.

Meseguer Yebra, J. (2015). "Las mejoras autonómicas en la regulación del derecho de acceso a la información pública”. Revista General de Derecho Administrativo, 38.

Moreno Molina, J. A. (2008). "La importancia de los principios generales de la contratación pública en la LCSP”. En J. A. Moreno Molina (Ed.), La Ley de Contratos del Sector Público y su aplicación por las Entidades Locales. Granada: CEMCl.

OECD. (2005). Public sector integrity. A framework for assessment. Paris: OECD.

OECD. (2009). OECD Principles for Integrity in Public Procurement. Paris: OECD. DOI: 10.1787/9789264056527-en.

Parycek, P., \& Sachs, M. (2010). "Open government-information flow in Web 2.0". European Journal of ePractice, 9 (march).

PricewaterhouseCoopers, \& Ecorys. (2013). Identifying and Reducing Corruption in Public Procurement in the EU. Development of a methodology to estimate the direct costs of corruption and other elements for an EUevaluation mechanism in the area of anti-corruption.

Ramírez-Alujas, Á. (2011). "Gobierno abierto y modernización de la gestión pública: tendencias actuales y el (inevitable) camino que viene. Reflexiones seminales”. Enfoques, 15(99-125).

Rose-Ackerman, S., \& Truex, R. (2012). Corruption and Policy Reform. Paper presented at the Copenhagen Consensus Project, Copenhagen. 
Sanmartín Mora, M. A. (2014). Contratación Abierta, ¿qué es?

Tschorne Berestesky, S. (2012). "Transparencia y control de la obra pública en Chile". En N. Dassen \& J. C. Veyra (Eds.), Gobierno Abierto y Transparencia Focalizada. Tendencias y desafíos para América Latina y el Caribe. Washington, D.C.: Banco Interamericano de Desarrollo.

United Nations. (2014). A world that counts. Mobilizing the data revolution for sustainable development.

Velasco Rico, C. (2014). “La cuestión competencial en la Ley 19/2013, de 9 de diciembre: ¿de qué margen disponen las CC.AA. en materia de transparencia, acceso a la información pública y buen gobierno?”. Revista Jurídica de Castilla y León, 33.

Villoria Mendieta, M. (2010). “La democratización de la administración pública: marco teórico”. En J. Ruiz-Huerta \& M. Villoria Mendieta (Eds.), Gobernanza democrática y fiscalidad.

Wirtz, B. W., \& Birkmeyer, S. (2015). “Open Government: Origin, Development, and Conceptual Perspectives". International Journal of Public Administration, 38(5), 381-396. DOI: 10.1080/01900692.2014.942735. 